\title{
Nutrición en pancreatitis aguda: nuevos conceptos para un viejo problema
}

\section{Nutrition in acute pancreatitis: new concepts for an old problem}

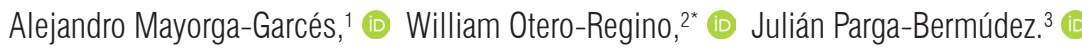

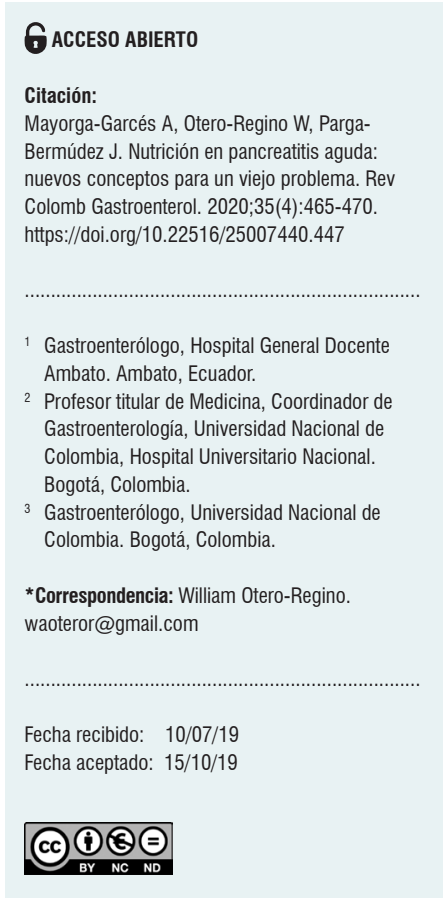

\begin{abstract}
Resumen
El manejo de la nutrición en pancreatitis aguda ha sido cuestión de debate. Durante muchos años el concepto de reposo pancreático fue generalizado y aceptado en el manejo de la pancreatitis aguda. Actualmente se conoce que la nutrición temprana permite mantener la integridad de la barrera intestinal, que previene la aparición de complicaciones infeccionas y se asocia con una menor estancia hospitalaria, menos complicaciones y un mejor pronóstico. En esta revisión se discuten las principales ventajas de la nutrición temprana en pancreatitis aguda, la seguridad de la misma y la vía de administración.
\end{abstract}

\section{Palabras clave}

Nutrición, reposo pancreático, sonda nasogástrica, sonda nasoyeyunal.

\begin{abstract}
Nutrition management in acute pancreatitis has been a matter of debate worldwide. For many years, the concept of pancreatic rest was widespread and accepted to treat acute pancreatitis. However, current knowledge of early nutrition allows maintaining the intestinal barrier's integrity, preventing the occurrence of infectious complications, which is associated with a shorter hospital stay, fewer complications, and better prognosis. This review presents the main advantages of early nutrition in acute pancreatitis, its safety, and the route of administration.
\end{abstract}

\section{Keywords}

Nutrition, Pancreatic rest, Nasogastric tube, Nasojejunal tube.

\section{INTRODUCCIÓN}

La pancreatitis aguda (PA) es uno de los trastornos gastrointestinales más frecuentes, sus principales etiologías son los cálculos biliares y el alcohol $(1,2)$.

Según los criterios de Atlanta, para su diagnóstico se requieren dos de los tres siguientes criterios: dolor abdominal compatible con PA, amilasa o lipasa elevados 3 veces sobre el límite superior normal, e imágenes características de PA demostradas por estudios de imagen $(3,4)$.
El $80 \%$ de los casos tiene un curso leve, mientras que un 20 $\%$ corresponde a pancreatitis moderadamente grave y grave. De estas, el $33 \%$ presentará una necrosis infectada, alcanzando una mortalidad de aproximadamente $15 \%$-35\%, siendo la sepsis la principal determinante de la misma. (5).

\section{CONSECUENCIAS DE LA RESTRICCIÓN DE LA DIETA}

En 1904 el médico ruso Iván Pávlov ganó el premio Nobel en Medicina por sus trabajos en animales, sobre la fisiología 
de la digestión y la respuesta a los estímulos sobre la secreción de distintas glándulas. Esto fue extrapolado por otros investigadores, quienes asumieron que suprimir el estímulo alimenticio evitaría las distintas fases de la secreción pancreática y, por consiguiente, la liberación de enzimas y mayor daño tisular, teoría que se mantuvo por varios años, incluso actualmente varios médicos ven con recelo administrar alimentos de manera temprana en la PA (6).

Los actuales conocimientos sobre la fisiopatología de la PA han demostrado que la producción de diferentes sustancias proinflamatorias, sumadas a la restricción alimenticia, conducen a una alteración en la motilidad intestinal y en la microbiota sapróbica, facilitando el sobrecrecimiento bacteriano y la alteración de la barrera intestinal, lo que permite la translocación bacteriana mediante los ganglios linfáticos hacia la circulación sistémica, donde las endotoxinas bacterianas provocan la aparición de sepsis y falla multiorgánica $(7,8)$. Esto se observa con mayor detalle en la Figura 1.

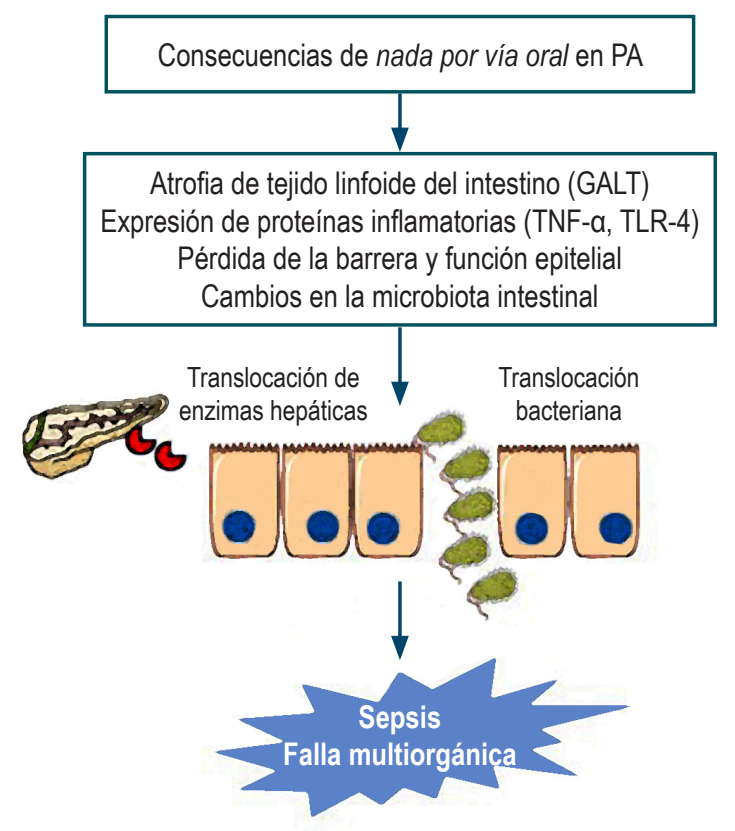

Figura 1. Consecuencias de la suspensión de la vía oral. GALT: tejido linfoide asociado con el intestino; TLR-4: receptor tipo toll-4; TNF- $\alpha$ : factor de necrosis tumoral alfa. Figura realizada por los autores.

\section{NUTRICIÓN TEMPRANA}

Uno de los pilares fundamentales en el tratamiento de la PA durante muchos años fue la restricción de alimentos o el llamado reposo digestivo, mientras "mejoraba" el cuadro de $\mathrm{PA}$, pues se consideraba que los estímulos generados por la alimentación favorecerían la liberación y activación de las enzimas pancreáticas, y generar mayor daño tisular (9). Actualmente, no existen bases que sustenten esta creencia y se ha demostrado en diferentes estudios que el inicio temprano de la nutrición enteral (es decir, en las primeras 24 a 48 horas de haber empezado el cuadro) mejora el balance nitrogenado y disminuye la incidencia de infecciones, la mortalidad y la estancia hospitalaria, y la estimulación pancreática exocrina es mínima (10-12).

Cabe resaltar el alto gasto metabólico producido durante una PA, especialmente en las pancreatitis graves, incrementa los requerimientos de distintos nutrientes encargados de mantener la homeostasis del sistema inmune y la regeneración tisular (13). Según las guías de la European Society for Clinical Nutrition and Metabolism (ESPEN), hasta un $80 \%$ de los casos de pancreatitis aguda grave tiene una pérdida de proteína de $40 \mathrm{~g} /$ día llevando hacia un balance nitrogenado negativo (14).

\section{NUTRICIÓN ENTERAL FRENTE A LA NUTRICIÓN PARENTERAL}

Desde hace aproximadamente 3 décadas se considera que la nutrición parenteral (NP) es la mejor vía de alimentación en PA, principalmente en casos graves, pese a que se conocían sus desventajas como un mayor riesgo de infección del catéter, alteración electrolítica, falla multiorgánica, costo y dificultad de colocación $(15,16)$. Además, se debe considerar la hiperglucemia, la cual está presente en más de la mitad de los casos de NP, y constituye un factor de riesgo adicional para infecciones y mortalidad $(17,18)$.

El conocimiento actual sobre el papel del intestino en la fisiopatología de la PA y la seguridad y tolerabilidad de la nutrición enteral (NE) han desplazado en uso de NP (19). En el metaanálisis de Al-Omran y colaboradores, el cual incluyó a 348 personas, demostró de manera significativa el beneficio de la NE en términos de mortalidad, infección, falla multiorgánica y necesidad de cirugía en comparación con la NP (20). Estos datos fueron corroborados con dos recientes metaanálisis, el primero incluyó a 348 personas y determinó una reducción significativa de la mortalidad y la falla multiorgánica a favor de la NE (21), y el segundo incluyó a 562 personas y determinó que hubo un menor riesgo de infección y necesidad de cirugía con el uso de la NE (22).

\section{INICIO DE LA NUTRICIÓN}

Recientemente, la Asociación Americana de Gastroenterología recomendó comenzar la dieta en las primeras 24 horas de iniciado el cuadro, puesto que favorece la integridad y funcionalidad intestinal, mantiene las uniones intercelulares y estimula las enzimas del borde en cepillo, evitando así la translocación bacteriana (23).

En el metaanálisis de Feng y colaboradores que incluyó a 1007 pacientes se determinó el beneficio de la NE en las 
primeras 48 horas, en términos de falla orgánica y desarrollo del síndrome de respuesta inflamatoria sistémica (SIRS), no encontró diferencia en relación con la mortalidad y la necrosis pancreática (24).

Una reciente revisión sistemática determinó que el inicio temprano de la NE en las primeras 48 horas redujo el riesgo de necrosis infectada, falla orgánica, necesidad de cirugía y mortalidad, comparado con NE tardía y NP (25).

El metaanálisis realizado por Qi y colaboradores determinó que la NE en las primeras 24 horas se asoció con una reducción de complicaciones infeccionas y falla multiorgánica en pancreatitis aguda grave, no se encontró beneficio en pancreatitis leves y moderadamente graves (26).

Una revisión sistemática, realizada en España concluyó que el mejor momento para iniciar la dieta es cuando existe la presencia de ruidos intestinales; además, utilizaron una dieta calórica completa, la cual fue bien tolerada, por lo que se logró reducir la estancia hospitalaria y se mejoró el curso de la PA (27).

\section{SONDA NASOGÁSTRICA (SNG) FRENTE A LA SONDA NASOYEYUNAL (SNY)}

Una reciente revisión de Cochrane demostró que no existen diferencias significativas entre el uso de la SNG y SNY, ambas lograron mantener la integridad de la barrea intestinal y la primera demostró ser mejor tolerada (28). En el metaanálisis de Chang y colaboradores no se encontraron diferencias entre el uso de SNG y SNY en términos de eficacia y seguridad (29).

El uso de SNY se reserva para aquellos casos de intolerancia a la SNG, obstrucción al tracto de salida gástrico, obstrucción duodenal y balance energético negativo con el uso de SNG (30). Otro beneficio que demostró el uso de la SNG fue la reducción del dolor, de la necesidad de opioides y de la intolerancia oral (31).

\section{COMPOSICIÓN DE LA DIETA}

No existe un consenso general sobre la composición ideal y el tipo de dieta, ya que la evidencia actual es limitada. En numerosos estudios se ha demostrado que aquellos que toleran la vía oral se beneficiaron de dietas bajas en grasa de composición sólida o líquida; esto actualmente supondría una ventaja, debido a que la práctica de ir modificando progresivamente la consistencia de la dieta produce un incremento innecesario en los días de estancia hospitalaria (32-35).

En el estudio realizado por Endo y colaboradores en 2018 no se encontró diferencia entre el uso de fórmulas elementales, semielementales y poliméricas (36). Larino-Noia y colaboradores, en un estudio realizado en España, no encontraron diferencias en la tolerancia a la dieta, iniciando la alimentación con una dieta sólida o dieta progresiva (37).

\section{USO DE SUPLEMENTOS}

Se ha propuesto el uso de distintos suplementos como probióticos, prebióticos o simbióticos, por su efecto sobre la microbiota y su papel protector, trófico y metabólico; y aunque se ha encontrado que el uso de estos podría asociarse con una disminución de la estancia hospitalaria, todavía no existe evidencia suficiente para recomendar su uso de manera rutinaria $(38,39)$.

Respecto al uso de aminoácidos como arginina y glutamina, los cuales en teoría podrían tener algún beneficio al mantener la integridad intestinal, las guías de la American Society for Parenteral and Enteral Nutrition (ASPEN) no recomiendan su uso rutinario (40).

Los ácidos grasos $\omega-3$, antioxidantes y vitaminas, cuya capacidad antinflamatoria podría suponer un beneficio en la PA, tampoco se han recomendado de manera rutinaria, puesto que se requieren más estudios que respalden su uso $(41,42)$. En la Tabla 1 se resumen las principales recomendaciones de la nutrición en PA.

Tabla 1. Principales recomendaciones del manejo nutricional en PA
Manejo

Reposo pancreático

NE frente a NP

Inicio de la

alimentación

SNG frente a SNY

Progresión de la

dieta

Composición de la No existe diferencia entre fórmulas

dieta

Uso de suplementos No se recomienda ninguno (probióticos, vitaminas, antioxidantes, aminoácidos, u otros)

Contraindicación

para la alimentación

Manejo del dolor

Ausencia de ruidos intestinales

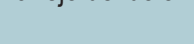

Los opioides continúan siendo la primera línea. La nutrición temprana disminuye el dolor y mejora la tolerancia oral
Tabla realizada por los autores. 


\section{CONCLUSIONES}

El concepto de reposo pancreático actualmente es obsoleto. El inicio temprano de la dieta no causa estimulación ni daño pancreático adicional. El efecto benéfico de la alimentación temprana sobre la barrera intestinal se asocia con una menor translocación bacteriana, menor riesgo de SIRS y menor mortalidad. La vía enteral es ampliamente superior a la parenteral. No existen diferencias entre el uso de SNG y SNY, y en caso de estar indicada, se prefiere la SNG por su facilidad de colocación. El dolor no es una indicación para suspender la dieta, por lo que es necesario optimizar la analgesia con el fin de continuar la alimentación, ya que la misma se asocia con una menor necesidad de opioides y disminución de la intensidad del dolor.

\section{Conflictos de interés}

Ninguno.

\section{REFERENCIAS}

1. Peery AF, Crockett SD, Murphy CC, Lund JL, Dellon ES, Williams JL, Jensen ET, Shaheen NJ, Barritt AS, Lieber SR, Kochar B, Barnes EL, Fan YC, Pate V, Galanko J, Baron TH, Sandler RS. Burden and Cost of Gastrointestinal, Liver, and Pancreatic Diseases in the United States: Update 2018. Gastroenterology. 2019;156(1): 254-272.e11. https://doi.org/10.1053/j.gastro.2018.08.063

2. Olson E, Perelman A, Birk JW. Acute management of pancreatitis: the key to best outcomes. Postgrad Med J. 2019;95(1124):328-333. https://doi.org/10.1136/postgradmedj-2018-136034

3. Tenner S, Baillie J, DeWitt J, Vege SS; American College of Gastroenterology. American College of Gastroenterology guideline: management of acute pancreatitis. Am J Gastroenterol. 2013;108(9):1400-15;1416. https://doi.org/10.1038/ajg.2013.218

4. Bollen TL. Acute pancreatitis: international classification and nomenclature. Clin Radiol. 2016;71(2):121-33. https://doi.org/10.1016/j.crad.2015.09.013

5. van Dijk SM, Hallensleben NDL, van Santvoort HC, Fockens P, van Goor H, Bruno MJ, Besselink MG; Dutch Pancreatitis Study Group. Acute pancreatitis: recent advances through randomised trials. Gut. 2017;66(11):2024-2032. https://doi.org/10.1136/gutjnl-2016-313595

6. Petrov MS, Pylypchuk RD, Emelyanov NV. Systematic review: nutritional support in acute pancreatitis. Alim Pharmacol Ther. 2008;28(6):704-712. https://doi.org/10.1111/j.1365-2036.2008.03786.x

7. Singh P, Garg PK. Pathophysiological mechanisms in acute pancreatitis: Current understanding. Indian J Gastroenterol. 2016;35(3):153-66. https://doi.org/10.1007/s12664-016-0647-y

8. Schietroma M, Pessia B, Carlei F, Mariani P, Sista F, Amicucci G. Intestinal permeability and systemic endotoxemia in patients with acute pancreatitis. Ann Ital Chir. 2016;87:138-44.

9. Gupta R, Patel K, Calder PC, Yaqoob P, Primrose JN, Johnson CD. A randomised clinical trial to assess the effect of total enteral and total parenteral nutritional support on metabolic, inflammatory and oxidative markers in patients with predicted severe acute pancreatitis (APACHE II $>$ or =6). Pancreatology. 2003;3(5):406-13. https://doi.org/10.1159/000073657

10. Ramanathan M, Aadam AA. Nutrition Management in Acute Pancreatitis. Nutr Clin Pract. 2019;34 Suppl 1:S7-S12. https://doi.org/10.1002/ncp.10386

11. Vaughn VM, Shuster D, Rogers MAM, Mann J, Conte ML, Saint S, Chopra V. Early Versus Delayed Feeding in Patients With Acute Pancreatitis: A Systematic Review. Ann Intern Med. 2017;166(12):883-892. https://doi.org/10.7326/M16-2533

12. Zhang D, Li H, Li Y, Qu L. Gut rest strategy and trophic feeding in the acute phase of critical illness with acute gastrointestinal injury. Nutr Res Rev. 2019;32(2):176-182. https://doi.org/10.1017/S0954422419000027

13. Pan LL, Li J, Shamoon M, Bhatia M, Sun J. Recent advances on nutrition in treatment of acute pancreatitis. Front Immunol. 2017;8:762. https://doi.org/10.3389/fimmu.2017.00762

14. Gianotti L, Meier R, Lobo DN, Bassi C, Dejong CH, Ockenga J, Irtun O, MacFie J; ESPEN. ESPEN guidelines on parenteral nutrition: pancreas. Clin Nutr. 2009;28(4):428-35. https://doi.org/10.1016/j.clnu.2009.04.003

15. Ziegler TR. Parenteral nutrition in the critically ill patient. N Engl J Med. 2009;361(11):1088-97. https://doi.org/10.1056/NEJMct0806956

16. Storck LJ, Imoberdorf R, Ballmer PE. Nutrition in Gastrointestinal Disease: Liver, Pancreatic, and Inflammatory Bowel Disease. J Clin Med. 2019;8(8). pii: E1098. https://doi.org/10.3390/jcm8081098

17. Pasquel FJ, Spiegelman R, McCauley M, Smiley D, Umpierrez D, Johnson R, Rhee M, Gatcliffe C, Lin E, Umpierrez E, Peng L, Umpierrez GE. Hyperglycemia during total parenteral nutrition: an important marker of poor outcome and mortality in hospitalized patients. Diabetes Care. 2010;33(4):739-741. https://doi.org/10.2337/dc09-1748

18. Petrov MS, Whelan K. Comparison of complications attributable to enteral and parenteral nutrition in predicted 
severe acute pancreatitis: a systematic review and metaanalysis. Br J Nutr. 2010;103(9):1287-95. https://doi.org/10.1017/S0007114510000887

19. Petrov MS. Gastric feeding and "gut rousing" in acute pancreatitis. Nutr Clin Pract. 2014;29(3):287-90. https://doi.org/10.1177/0884533614528986

20. Al-Omran M, Albalawi ZH, Tashkandi MF, Al-Ansary LA. Enteral versus parenteral nutrition for acute pancreatitis. Cochrane Database Syst Rev. 2010;(1):Cd002837. https://doi.org/10.1002/14651858.CD002837.pub2

21. Yao H, He C, Deng L, Liao G. Enteral versus parenteral nutrition in critically ill patients with severe pancreatitis: a meta-analysis. Eur J Clin Nutr. 2018;72(1):66-68. https://doi.org/10.1038/ejcn.2017.139

22. Wu P, Li L, Sun W. Efficacy comparisons of enteral nutrition and parenteral nutrition in patients with severe acute pancreatitis: a meta- analysis from randomized controlled trials. Biosci Rep. 2018;38(6):pii: BSR20181515. https://doi.org/10.1042/BSR20181515

23. Crockett SD, Wani S, Gardner TB, Falck-Ytter Y, Barkun AN; American Gastroenterological Association Institute Clinical Guidelines Committee. American Gastroenterological Association Institute Guideline on Initial Management of Acute Pancreatitis. Gastroenterology. 2018;154(4):1096-1101. https://doi.org/10.1053/j.gastro.2018.01.032

24. Feng P, He C, Liao G, Chen Y. Early enteral nutrition versus delayed enteral nutrition in acute pancreatitis: A PRISMA compliant systematic review and meta-analysis. Medicine (Baltimore). 2017;96(46):e8648. https://doi.org/10.1097/MD.0000000000008648

25. Song J, Zhong Y, Lu X, Kang X, Wang Y, Guo W, Liu J, Yang Y, Pei L. Enteral nutrition provided within 48 hours after admission in severe acute pancreatitis: a systematic review and meta-analysis. Medicine (Baltimore). 2018;97(34):e11871. https://doi.org/10.1097/MD.0000000000011871

26. Qi D, Yu B, Huang J, Peng M. Meta-Analysis of Early Enteral Nutrition Provided Within 24 Hours of Admission on Clinical Outcomes in Acute Pancreatitis. JPEN J Parenter Enteral Nutr. 2018;42(7):1139-1147. https://doi.org/10.1002/jpen.1139

27. Valverde-López F, Wilcox CM, Redondo-Cerezo E. Evaluation and management of acute pancreatitis in Spain. Gastroenterol Hepatol. 2018;41(10):618-628. https://doi.org/10.1016/j.gastrohep.2018.06.012

28. Moggia E, Koti R, Belgaumkar AP, Fazio F, Pereira SP, Davidson BR, Gurusamy KS. Pharmacological interventions for acute pancreatitis. Cochrane Database Syst Rev. 2017;4(4):CD011384. https://doi.org/10.1002/14651858.CD011384.pub2

29. Chang YS, Fu HQ Xiao YM, Liu JC. Nasogastric or nasojejunal feeding in predicted severe acute pancreatitis: a metaanalysis. Crit Care. 2013;17(3):R118.

https://doi.org/10.1186/cc12790
30. Faghih M, Fan C, Singh VK. New Advances in the Treatment of Acute Pancreatitis. Curr Treat Options Gastroenterol. 2019;17(1):146-160. https://doi.org/10.1007/s11938-019-00223-8

31. Petrov MS, McIlroy K, Grayson L, Phillips AR, Windsor JA. Early nasogastric tube feeding versus nil per os in mild to moderate acute pancreatitis: a randomized controlled trial. Clin Nutr. 2013;32(5): 697-703. https://doi.org/10.1016/j.clnu.2012.12.011

32. Li J, Chen J, Tang W. The consensus of integrative diagnosis and treatment of acute pancreatitis-2017. J Evid Based Med. 2019;12(1):76-88. https://doi.org/10.1111/jebm.12342

33. Vege SS, DiMagno MJ, Forsmark CE, Martel M, Barkun AN. Initial medical treatment of acute pancreatitis: American Gastroenterological Association Institute technical review. Gastroenterology. 2018;154(4):1103-1139. https://doi.org/10.1053/j.gastro.2018.01.031

34. Lodewijkx PJ, Besselink MG, Witteman BJ, Schepers NJ, Gooszen HG, van Santvoort HC, Bakker OJ; Dutch Pancreatitis Study Group. Nutrition in acute pancreatitis: a critical review. Expert Rev Gastroenterol Hepatol. 2016;10(5):571-80. https://doi.org/10.1586/17474124.2016.1141048

35. Roberts KM, Nahikian-Nelms M, Ukleja A, Lara LF. Nutritional Aspects of Acute Pancreatitis. Gastroenterol Clin North Am. 2018;47(1):77-94. https://doi.org/10.1016/j.gtc.2017.10.002

36. Endo A, Shiraishi A, Fushimi K, Murata K, Otomo Y. Comparative effectiveness of elemental formula in the early enteral nutrition manage- ment of acute pancreatitis: a retrospective cohort study. Ann Intensive Care. 2018;8(1):69. https://doi.org/10.1186/s13613-018-0414-6

37. Lariño-Noia J, Lindkvist B, Iglesias-García J, Seijo-Ríos S, Iglesias-Canle J, Domínguez-Muñoz JE. Early and/ or immediately full caloric diet versus standard refeeding in mild acute pancreatitis: a randomized open-label trial. Pancreatology. 2014;14(3):167-73. https://doi.org/10.1016/j.pan.2014.02.008

38. Tian X, Pi YP, Liu XL, Chen H, Chen WQ. Supplemented Use of Pre-, Pro-, and Synbiotics in Severe Acute Pancreatitis: An Updated Systematic Review and MetaAnalysis of 13 Randomized Controlled Trials. Front Pharmacol. 2018;9:690. https://doi.org/10.3389/fphar.2018.00690

39. Ballesteros Pomar MD, González Arnaiz E. Role of prebiotics and probiotics in the functionality of the microbiota in the patients receiving enteral nutrition. Nutr Hosp. 2018;35(Spec no2):18-26. https://doi.org/10.20960/nh.1956

40. McClave SA, Taylor BE, Martindale RG, Warren MM, Johnson DR, Braunschweig C, McCarthy MS, Davanos E, Rice TW, Cresci GA, Gervasio JM, Sacks GS, Roberts PR, Compher C; Society of Critical Care Medicine; American Society for Parenteral and Enteral Nutrition. Guidelines 
for the Provision and Assessment of Nutrition Support Therapy in the Adult Critically Ill Patient: Society of Critical Care Medicine (SCCM) and American Society for Parenteral and Enteral Nutrition (A.S.P.E.N.). JPEN J Parenter Enteral Nutr. 2016;40(2):159-211. https://doi.org/10.1177/0148607115621863

41. Lei QC, Wang XY, Xia XF, Zheng HZ, Bi JC, Tian F, Li $\mathrm{N}$. The role of omega- 3 fatty acids in acute pancreatitis: a meta-analysis of randomized controlled trials. Nutrients. 2015;7(4):2261-73.

https://doi.org/10.3390/nu7042261

42. Jeurnink SM, Nijs MM, Prins HA, Greving JP, Siersema PD. Antioxidants as a treatment for acute pancreatitis: A meta-analysis. Pancreatology. 2015;15(3):203-8.

https://doi.org/10.1016/j.pan.2015.03.009 